\title{
Applications of fixed point results for cyclic Boyd-Wong type generalized $F-\psi$-contractions to dynamic programming
}

\author{
Deepak Singh $^{\mathrm{a}}$, Varsha Chauhan ${ }^{\mathrm{b}}$, Poom Kumam ${ }^{\mathrm{c}, \mathrm{d}, \mathrm{e}, *}$, Vishal Joshi ${ }^{\mathrm{f}}$, Phatiphat Thounthong ${ }^{\mathrm{g}, \mathrm{h}}$ \\ ${ }^{a}$ Department of Applied Sciences, NITTTR, Under Ministry of HRD, Govt. of India, Bhopal, (M.P.), 462002 India. \\ ${ }^{b}$ Department of Mathematics, NRI Institute of Research \& Technology, Bhopal M.P, India. \\ ${ }^{c}$ KMUTTFixed Point Research Laboratory, Department of Mathematics, Faculty of Science, King Mongkut's University of Technology \\ Thonburi (KMUTT), 126 Pracha-Uthit Road, Bang Mod, Thung Khru, Bangkok 10140, Thailand. \\ ${ }^{d}$ KMUTT-Fixed Point Theory and Applications Research Group, Theoretical and Computational Science Center (TaCS), Science \\ Laboratory Building, Faculty of Science King Mongkut's University of Technology Thonburi (KMUTT), 126 Pracha Uthit Road, Bang \\ Mod, Thung Khru, Bangkok 10140, Thailand. \\ ${ }^{e}$ Department of Medical Research, China Medical University Hospital, China Medical University, Taichung 40402, Taiwan. \\ ${ }^{f}$ Department of Mathematics, Jabalpur Engineering College, Jabalpur (M.P.), India. \\ ${ }^{g}$ Renewable Energy Research Centre, King Mongkut's University of Technology North Bangkok (KMUTNB), Wongsawang, Bangsue, \\ Bangkok 10800, Thailand. \\ ${ }^{h}$ Department of Teacher Training in Electrical Engineering, Faculty of Technical Education, King Mongkut's University of Technology \\ North Bangkok (KMUTNB), Wongsawang, Bangsue, Bangkok 10800, Thailand.
}

\begin{abstract}
Recently, Piri et al. [H. Piri, P. Kumam, Fixed Point Theory Appl., 2014 (2014), 11 pages] refined the result of Wardowski [D. Wardowski, Fixed Point Theory Appl., 2012 (2012), 6 pages] by launching some weaker conditions on the self-map regarding a complete metric space and over the mapping F. In the article, we inaugurate Boyd-Wong type generalized F- $\psi$-contraction and prove some new fixed point results in partial metric spaces, also we deduce fixed point results involving cyclic BoydWong type generalized F- $\psi$-contraction in the same setup. These results substantially generalize and improve the corresponding theorems contained in Wardowski ([D. Wardowski, Fixed Point Theory Appl., 2012 (2012), 6 pages] [D. Wardowski, N. Van Dung, Demonstr. Math., 47 (2014), 146-155]), Matthews [S. G. Matthews, Papers on general topology and applications, Flushing, NY, (1992), 183-197, Ann. New York Acad. Sci., New York Acad. Sci., New York, 728 (1994)], and others. The paper includes two applications and some illustrative examples to highlight the realized improvements. (c)2017 all rights reserved.
\end{abstract}

Keywords: Fixed point, partial metric spaces, F-contraction, dynamic programming. 2010 MSC: 47H10, 54H25.

\section{Introduction and preliminaries}

The notion of partial metric space, departs from the usual metric spaces due to removing the assumption of self-distance. In other words, in partial metric space self-distance needs not to be zero. This

\footnotetext{
*Corresponding author

Email addresses: pdk.singh1002@gmail.com (Deepak Singh), varsha18chauhan@gmail.com (Varsha Chauhan), poom.kum@kmutt.ac.th (Poom Kumam), joshinvishal76@gmail.com (Vishal Joshi), phtt@kmutnb.ac.th (Phatiphat Thounthong)
}

doi:10.22436/jmcs.017.02.02 
interesting distance function is introduced by Matthews [9]. A motivation behind introducing the concept of a partial metric space was to present an altered version of a Banach contraction principle, more convenient to solve certain problems arising in computer science. Valero [20], Oltra and Valero [12] and Altun et al. [3] gave some further generalization of the results in [9]. Romaguera introduced in [15] the notion of a 0-Cauchy sequence in a partial metric space and of a 0-complete partial metric space.

In recent times, Romaguera [16] obtained the fixed point theorems of Boyd-Wong type due to Boyd et al. [5]. Further, Abbas et al. [1] extended the result of Romaguera [16] to cyclic mappings and proved the result, taking $\phi \in \Phi$ as a continuous map. Most recently, Aydi et al. [4] presented the result of Abbas et al. [1] for a shaky assumptions over $\phi \in \Phi$. Ones can find new results on the fixed point theory and application in partial metric spaces in $[2,6,11]$.

Recently, Wardowski [21] introduced a new contraction called F-contraction and proved a fixed point theorem as a generalization of the Banach contraction principle. Secelean [17] characterized a large class of functions by renewal the condition (F2') instead of the condition (F2) in the definition of F-contraction presented by Wardowski [21]. Very recently, Piri et al. [14] improved the result of Secelean [17] by replacing condition (F3') instead of the condition (F3). Moreover, Hussain [7] extended Wardowski fixed point results for F-contractions in complete metric spaces.

In the sequel, $\mathbb{R}, \mathbb{N}$ and $\mathbb{N}^{*}$ will represent the set of all real numbers, natural numbers and positive integers, respectively. Some elementary definitions and fundamental results of partial metric space and F-contraction are borrowed, which are essential in our subsequent discussion.

Definition 1.1 ([9]). A partial metric on a nonempty set $X$ is a function $p: X \times X \rightarrow[0, \infty)$ such that for all $x, y, z \in X$

(p1) $x=y$, iff $p(x, x)=p(x, y)=p(y, y)$;

(p2) $p(x, x) \leqslant p(x, y)$;

(p3) $p(x, y)=p(y, x)$;

(p4) $p(x, y) \leqslant p(x, z)+p(z, y)-p(z, z)$.

The function $p$ is called a partial metric on $X$ and the pair $(X, p)$ is called a partial metric space.

Supposing that $p$ is a partial metric on $X$, at that time the function $d_{p}: X \times X \rightarrow[0, \infty)$ given by

$$
d_{p}(x, y)=2 p(x, y)-p(x, x)-p(y, y),
$$

is a metric on $X$.

Example 1.2 (see, e.g., [9]). Let $X=\{[a, b]: a, b \in \mathbb{R}, a \leqslant b\}$, and define $p([a, b],[c, d])=\max \{b, d\}-$ $\min \{a, c\}$. Then $(X, p)$ is a partial metric space.

Each partial metric $p$ on $X$ generates a $T_{0}$ topology $\tau_{p}$ on $X$, that carries as a base the class of open $p$-balls $\left\{B_{p}(x, \epsilon), x \in X, \epsilon>0\right\}$, where $B_{p}(x, \epsilon)=\{y \in X: p(x, y)<p(x, x)+\epsilon\}$ for all $x \in X$ and $\epsilon>0$.

Definition $1.3([9,12,15])$. Let $(X, p)$ be a partial metric space. Then

(i) a sequence $\left\{x_{n}\right\}$ converges to a point $x \in X$, if and only if $p(x, x)=\lim _{n \rightarrow+\infty} p\left(x, x_{n}\right)$;

(ii) a sequence $\left\{x_{n}\right\}$ is called a Cauchy sequence, if $\lim _{n, m \rightarrow+\infty} p\left(x_{n}, x_{m}\right)$ exists and is finite;

(iii) $(X, p)$ is said to be complete, if every Cauchy sequence $\left\{x_{n}\right\}$ in $X$ converges to some $x \in X$ (with respect to $\left.\tau_{p}\right)$ such that $p(x, x)=\lim _{n, m \rightarrow+\infty} p\left(x_{n}, x_{m}\right)$;

(iv) a sequence $\left\{x_{n}\right\}$ in $(X, p)$ is called 0-Cauchy sequence, if $\lim _{n, m \rightarrow+\infty} p\left(x_{n}, x_{m}\right)=0$. The space $(X, p)$ 
is said to be 0 -complete whenever every 0 -Cauchy sequence in $X$ converges with respect to $\tau_{p}$ to a point $x \in X$ such that $p(x, x)=0$.

Lemma 1.4 $([9,12,15])$. Let $(X, p)$ be a partial metric space. Then

1. $\left\{x_{n}\right\}$ is a Cauchy sequence in $(X, p)$, if and only if it is a Cauchy sequence in the metric space $\left(X, d_{p}\right)$.

2. $(X, p)$ is complete, if and only if the metric space $\left(X, d_{p}\right)$ is complete. Furthermore, $\lim _{n \rightarrow+\infty} d_{p}\left(x_{n}, X\right)=0$, if and only if $p(x, x)=\lim _{n \rightarrow+\infty} p\left(x_{n}, x\right)=\lim _{n, m \rightarrow+\infty} p\left(x_{n}, x_{m}\right)$.

3. Every 0 -Cauchy sequence in $(X, p)$ is Cauchy in $(X, d)$.

4. If $(\mathrm{X}, \mathrm{p})$ is complete then it is 0 -complete.

Lemma 1.5 ([9]). Let $(X, p)$ be a partial metric space. Then

1. if $p(x, y)=0$, then $x=y$. But if $x=y$, then $p(x, y)$ may not be zero;

2. if $x \neq y$, then $p(x, y)>0$.

Lemma 1.6 ([13]). Let $x_{n} \rightarrow z$ as $n \rightarrow \infty$ in a partial metric space $(X, p)$ where $p(z, z)=0$. Then $\lim _{n \rightarrow \infty} p\left(x_{n}, y\right)=$ $\mathrm{p}(z, \mathrm{y})$ for every $\mathrm{y} \in \mathrm{X}$.

Let $\Phi$ be the set of functions $\phi:[0, \infty) \rightarrow[0, \infty)$ such that

1. $\phi$ is upper semi-continuous (i.e. for any sequence $\left\{t_{n}\right\}$ in $[0, \infty)$ such that $t_{n} \rightarrow t$ as $n \rightarrow \infty$, we have $\left.\lim \sup \phi\left(t_{n}\right) \leqslant \phi(t)\right)$;

$n \rightarrow \infty$

2. $\phi(t)<t$, for each $t>0$.

Let $\Psi$ denote the set of all decreasing functions $\psi:(0, \infty) \rightarrow(0, \infty)$.

Recently, Romaguera [16] developed the following fixed point theorem of Boyd-Wong type [5].

Theorem 1.7. Let $(X, p)$ be a complete partial metric space along with $T: X \rightarrow X$ being a map such that for all $x, y \in X$

$$
p(T x, T y) \leqslant \phi(M(x, y))
$$

where

$$
M(x, y)=\max \left\{p(x, y), p(x, T x), p(y, T y), \frac{1}{2}[p(x, T y)+p(y, T x)]\right\},
$$

and $\phi \in \Phi$. Then, T has a unique fixed point.

Following definition is due to Wardowski [21].

Definition 1.8. Let $F: \mathbb{R}^{+} \rightarrow \mathbb{R}$ be a mapping satisfying:

(F1) $F$ is strictly increasing, that is, for all $\alpha, \beta \in \mathbb{R}^{+}$, if $\alpha<\beta$ then $F(\alpha)<F(\beta)$;

(F2) for each sequence $\left\{\alpha_{n}\right\}$ of positive numbers $\lim _{n \rightarrow \infty} \alpha_{n}=0$, if and only if $\lim _{n \rightarrow \infty} F\left(\alpha_{n}\right)=-\infty$;

(F3) there exists $k \in(0,1)$ such that $\lim _{\alpha \rightarrow 0^{+}} \alpha^{k} \mathrm{~F}(\alpha)=0$.

We denote the set of all functions satisfying (F1)-(F3) by $\digamma$. In [21], Wardowski defined F-contraction as follows:

Let $(X, \rho)$ be a metric space, then the mapping $T: X \rightarrow X$ is said to be an F-contraction, if there exist $F \in \digamma$ and $\tau>0$ such that for all $x, y \in X, \rho(T x, T y)>0$, we have

$$
\tau+F(\rho(T x, T y)) \leqslant F(\rho(x, y)) .
$$

On the other hand, Secelean [17] reintegrated the condition (F2) by more elementary condition (F2'). 
$\left(F 2^{\prime}\right) \inf F=-\infty$,

or, also by

$\left(F 2^{\prime \prime}\right)$ there exists a sequence $\left\{\alpha_{n}\right\}_{n=1}^{\infty}$ of positive real numbers such that $\lim _{n \rightarrow \infty} F\left(\alpha_{n}\right)=-\infty$.

Most recently, Piri et al. [14] used the following condition (F3') instead of (F3).

$\left(\mathrm{F}^{\prime}\right) \mathrm{F}$ is continuous on $(0, \infty)$.

We denote the set of all functions satisfying (F1), (F2') and (F3') by $\Delta_{\mathrm{F}}$.

Our work is devoted to introduce the Boyd-Wong type generalized F- $\psi$-contraction, by integrating the ideas of Wardowski [21] and Romaguera [16]. Moreover, the Boyd-Wong type cyclic generalized F- $\psi-$ contraction by combining the essence of Wardowski [21] and Aydi et al. [4] is also established. Invoking aforesaid developments some new fixed point theorems in complete partial metric space are proved.

\section{Main results}

\subsection{Results on Boyd-Wong type generalized $\mathrm{F}-\psi-$-contractions in partial metric space}

In this section we present our essential results. For this, we introduce the following definition.

Definition 2.1. Let $(X, p)$ be a partial metric space and $T: X \rightarrow X$ be a mapping. Then $T$ is said to be a Boyd-Wong type generalized $F-\psi$-contraction, if there exist $F \in \Delta_{F}, \psi \in \Psi$ and $\phi \in \Phi$ such that for all $x, y \in X$ with $p(T x, T y)>0$,

$$
\psi(p(x, y))+F(p(T x, T y)) \leqslant F(\phi(\mathbb{M}(x, y)))
$$

where

$$
\mathbb{M}(x, y)=\max \left\{p(x, y), p(x, T x), p(y, T y), \frac{1}{2}[p(x, T y)+p(y, T x)]\right\}
$$

One of our main results is as follows.

Theorem 2.2. Let $(\mathrm{X}, \mathrm{p})$ be a 0-complete partial metric space and $\mathrm{T}: \mathrm{X} \rightarrow \mathrm{X}$ be a Boyd-Wong type generalized $\mathrm{F}-\mathrm{\psi}$-contraction on $\mathrm{X}$. Then $\mathrm{T}$ has a unique fixed point $\mathrm{u} \in \mathrm{X}$, moreover $\mathrm{p}(\mathrm{u}, \mathrm{u})=0$.

Proof. For an arbitrary $x \in X$, we construct a sequence $\left\{x_{n}\right\}$ in the following way:

$$
x=x_{0} \text { and } x_{n+1}=T x_{n}, \text { for all } n \in \mathbb{N}^{*} .
$$

If there exists $n_{0} \in \mathbb{N}^{*}$ such that $x_{n_{0}}=x_{n_{0}+1}$, then $x_{n_{0}}$ is the desired fixed point of $T$ which completes the proof. Consequently, we assume that $x_{n} \neq x_{n+1}$ or $p\left(T x_{n-1}, T x_{n}\right)>0$ for all $n \in \mathbb{N}^{*}$; then it follows from (2.1) that

$$
\psi\left(p\left(x_{n-1}, x_{n}\right)\right)+F\left(p\left(x_{n}, x_{n+1}\right)\right)=\psi\left(p\left(x_{n-1}, x_{n}\right)\right)+F\left(p\left(T x_{n-1}, T x_{n}\right)\right) \leqslant F\left(\phi\left(\mathbb{M}\left(x_{n-1}, x_{n}\right)\right)\right),
$$

where

$$
\begin{aligned}
\mathbb{M}\left(x_{n-1}, x_{n}\right) & =\max \left\{p\left(x_{n-1}, x_{n}\right), p\left(x_{n-1}, x_{n}\right), p\left(x_{n}, x_{n+1}\right), \frac{1}{2}\left[p\left(x_{n-1}, x_{n+1}\right)+p\left(x_{n}, x_{n}\right)\right]\right\} \\
& =\max \left\{p\left(x_{n-1}, x_{n}\right), p\left(x_{n}, x_{n+1}\right)\right\} .
\end{aligned}
$$

If for some $n \in \mathbb{N}^{*}, \mathbb{M}\left(x_{n-1}, x_{n}\right)=p\left(x_{n}, x_{n+1}\right)$, then taking (2.3) into account, we obtain that

$$
\psi\left(p\left(x_{n-1}, x_{n}\right)\right)+F\left(p\left(x_{n}, x_{n+1}\right)\right) \leqslant F\left(\phi\left(p\left(x_{n}, x_{n+1}\right)\right)\right) .
$$


On using the property of $\phi$ and from (F1), we acquire that

$$
\psi\left(p\left(x_{n-1}, x_{n}\right)\right)+F\left(p\left(x_{n}, x_{n+1}\right)\right)<F\left(p\left(x_{n}, x_{n+1}\right)\right) .
$$

Since, $\psi\left(p\left(x_{n-1}, x_{n}\right)\right)>0$, which gives a contradiction, yielding thereby

$$
\mathbb{M}\left(x_{n-1}, x_{n}\right)=p\left(x_{n-1}, x_{n}\right), \quad \forall n \in \mathbb{N}^{*} .
$$

Therefore from (2.3) and by the property of $F, \phi$ and $\psi$, we arrive at

$$
\begin{aligned}
& F\left(p\left(x_{n}, x_{n+1}\right)\right) \leqslant F\left(\phi\left(p\left(x_{n-1}, x_{n}\right)\right)\right)-\psi\left(p\left(x_{n-1}, x_{n}\right)\right) \leqslant F\left(\phi\left(p\left(x_{n-1}, x_{n}\right)\right)\right), \\
& F\left(p\left(x_{n}, x_{n+1}\right)\right)<F\left(p\left(x_{n-1}, x_{n}\right)\right) .
\end{aligned}
$$

It follows from the above inequality and the property (F1) that

$$
p\left(x_{n}, x_{n+1}\right)<p\left(x_{n-1}, x_{n}\right), \text { for all } n \in \mathbb{N}^{*} .
$$

Thus $\left\{p\left(x_{n}, x_{n+1}\right)\right\}$ is a decreasing sequence of positive real numbers. Consequently from (2.4), we have

$$
\begin{aligned}
F\left(p\left(x_{n}, x_{n+1}\right)\right) & <F\left(p\left(x_{n-1}, x_{n}\right)\right)-\psi\left(p\left(x_{n-1}, x_{n}\right)\right) \\
& <F\left(p\left(x_{n-2}, x_{n-1}\right)\right)-\psi\left(p\left(x_{n-2}, x_{n-1}\right)\right)-\psi\left(p\left(x_{n-1}, x_{n}\right)\right) .
\end{aligned}
$$

As $\psi$ is a decreasing function, we get

$$
F\left(p\left(x_{n}, x_{n+1}\right)\right)<F\left(p\left(x_{n-2}, x_{n-1}\right)\right)-2 \psi\left(p\left(x_{n-2}, x_{n-1}\right)\right) .
$$

It follows by successive application that

$$
F\left(p\left(x_{n}, x_{n+1}\right)\right)<F\left(p\left(x_{0}, x_{1}\right)\right)-n \psi\left(p\left(x_{0}, x_{1}\right)\right) .
$$

Since, $F \in \Delta_{F}$, letting the limit as $n \rightarrow \infty$ in (2.5) we must have

$$
\lim _{n \rightarrow \infty} F\left(p\left(x_{n}, x_{n+1}\right)\right)=-\infty \Longleftrightarrow \lim _{n \rightarrow \infty} p\left(x_{n}, x_{n+1}\right)=0 .
$$

Further, by (p2) we have the following equality

$$
\lim _{n \rightarrow \infty} p\left(x_{n}, x_{n}\right)=0
$$

Next, we shall show that $\left\{x_{\mathfrak{n}}\right\}_{\mathfrak{n}=1}^{\infty}$ is a Cauchy sequence in $X$. Suppose, to the contrary, that $\left\{x_{\mathfrak{n}}\right\}_{\mathfrak{n}=1}^{\infty}$ is not a Cauchy sequence in a complete partial metric space $(X, p)$. Then there exist $\epsilon>0$ and two sub-sequences $\left\{x_{n(k)}\right\}$ and $\left\{x_{m(k)}\right\}$ of $\left\{x_{n}\right\}_{n=1}^{\infty}$ such that, $n(k)>m(k) \geqslant k$ and

$$
p\left(x_{m(k)}, x_{n(k)}\right) \geqslant \epsilon
$$

which yields

$$
p\left(x_{m}(k), x_{n}(k)-1\right)<\epsilon .
$$

Utilizing the property (p4) and inequality (2.8), we acquire that

$$
\begin{aligned}
\epsilon & \leqslant p\left(x_{m(k)}, x_{n(k)}\right) \leqslant p\left(x_{m(k)}, x_{m(k)+1}\right)+p\left(x_{m(k)+1}, x_{n(k)}\right)-p\left(x_{m(k)+1}, x_{m(k)+1}\right) \\
& \leqslant p\left(x_{m(k)}, x_{m(k)+1}\right)+p\left(x_{m(k)+1}, x_{m(k)}\right)+p\left(x_{m(k)}, x_{n(k)}\right)-p\left(x_{m(k)}, x_{m(k)}\right) \\
& \leqslant 2 p\left(x_{m(k)}, x_{m(k)+1}\right)+p\left(x_{m(k)}, x_{n(k)}\right) \\
& \leqslant 2 p\left(x_{m(k)}, x_{m(k)+1}\right)+p\left(x_{m(k)}, x_{n(k)-1}\right)+p\left(x_{n(k)-1}, x_{n(k)}\right)-p\left(x_{n(k)-1}, x_{n(k)-1}\right) \\
& \leqslant 2 p\left(x_{m(k)}, x_{m(k)+1}\right)+p\left(x_{m(k)}, x_{n(k)-1}\right)+p\left(x_{n(k)-1}, x_{n(k)}\right) \\
& \leqslant 2 p\left(x_{m(k)}, x_{m(k)+1}\right)+\epsilon+p\left(x_{n(k)-1}, x_{n(k)}\right),
\end{aligned}
$$


which on making $k \rightarrow \infty$, reduces to

$$
\lim _{k \rightarrow \infty} p\left(x_{m(k)}, x_{n(k)}\right)=\epsilon .
$$

Moreover, from (p4), (2.6), (2.7) and (2.9), one can easily get

$$
\begin{aligned}
& \lim _{k \rightarrow \infty} p\left(x_{m(k)}, x_{n(k)+1}\right)=\epsilon, \\
& \lim _{k \rightarrow \infty} p\left(x_{m(k)+1}, x_{n(k)}\right)=\epsilon,
\end{aligned}
$$

and

$$
\lim _{k \rightarrow \infty} p\left(x_{m(k)+1}, x_{n(k)+1}\right)=\epsilon .
$$

Also from (2.7) there exists a natural number $n_{0} \in \mathbb{N}$ such that

$$
\mathrm{p}\left(\mathrm{x}_{\mathrm{n}(\mathrm{k})}, \mathrm{x}_{\mathrm{n}(\mathrm{k})+1}\right)=\frac{\epsilon}{4} \text { and } \mathrm{p}\left(\mathrm{x}_{\mathrm{m}(\mathrm{k})}, \mathrm{x}_{\mathrm{m}(\mathrm{k})+1}\right)=\frac{\epsilon}{4},
$$

for all $n, m \geqslant n_{0}$. Now we claim that

$$
p\left(T x_{n(k)}, T x_{m(k)}\right)=p\left(x_{n(k)+1}, x_{m(k)+1}\right)>0 .
$$

On the contrary assume that, $p\left(x_{n(k)+1}, x_{m(k)+1}\right)=0$. Then

$$
\begin{aligned}
\epsilon & \leqslant p\left(x_{n(k)}, x_{m(k)}\right) \leqslant p\left(x_{n(k)}, x_{n(k)+1}\right)+p\left(x_{n(k)+1}, x_{m(k)}\right)-p\left(x_{n(k)+1}, x_{n(k)+1}\right) \\
& \leqslant p\left(x_{n(k)}, x_{n(k)+1}\right)+p\left(x_{n(k)+1}, x_{m(k)+1}\right)+p\left(x_{m(k)+1}, x_{m(k)}\right)-p\left(x_{m(k)+1}, x_{m(k)+1}\right) \\
& \leqslant p\left(x_{n(k)}, x_{n(k)+1}\right)+p\left(x_{n(k)+1}, x_{m(k)+1}\right)+p\left(x_{m(k)+1}, x_{m(k)}\right) \\
& \leqslant \frac{\epsilon}{4}+0+\frac{\epsilon}{4}=\frac{\epsilon}{2},
\end{aligned}
$$

which yields a contradiction, thus (2.11) holds. Then it follows by the contractive condition (1.1) and the property of $\psi$ that

$$
\begin{aligned}
\psi\left(p\left(x_{n(k)}, x_{m(k)}\right)\right)+F\left(p\left(x_{n(k)+1}, x_{m(k)+1}\right)\right) & =\psi\left(p\left(x_{n(k)}, x_{m(k)}\right)\right)+F\left(p\left(T x_{n(k)}, T x_{m(k)}\right)\right) \\
& \leqslant F\left(\phi\left(\mathbb{M}\left(x_{n(k)}, x_{m(k)}\right)\right)\right), \quad \text { i.e., } \\
F\left(p\left(x_{n(k)+1}, x_{m(k)+1}\right)\right) & \leqslant F\left(\phi\left(\mathbb{M}\left(x_{n(k)}, x_{m(k)}\right)\right)\right) .
\end{aligned}
$$

By the definition of $\mathbb{M}(x, y),(2.9),(2.10)$ and after routine calculations, we have

$$
\lim _{k \rightarrow \infty} \mathbb{M}\left(x_{n(k)}, x_{m(k)}\right)=\epsilon .
$$

Letting $k \rightarrow \infty$ in (2.12) and taking into account (2.6), (2.9), (2.10), (2.13), property (F3') and upper semicontinuity of $\phi$, we find that $F(\epsilon) \leqslant F(\phi(\epsilon))<F(\epsilon)$, which gives a contradiction. Thus, we conclude that $\lim _{n, m \rightarrow \infty} p\left(x_{n}, x_{m}\right)=0$, that is, the sequence $\left\{x_{n}\right\}_{n=1}^{\infty}$ is a 0 -Cauchy sequence. Therefore, the 0 -completeness of $X$ ensures that there exists a point $u \in X$ such that

$$
\lim _{n, m \rightarrow \infty} p\left(x_{n}, x_{m}\right)=\lim _{n \rightarrow \infty} p\left(x_{n}, u\right)=p(u, u)=0 .
$$

We will prove that $u$ is the fixed point of $T$. Since $T$ is continuous, therefore by using (2.14) and Lemma 1.6, we have

$$
p(u, T u)=\lim _{n \rightarrow \infty} p\left(x_{n}, T u\right)=\lim _{n \rightarrow \infty} p\left(T x_{n-1}, T u\right)=p(T u, T u)
$$


Next, we show that $p(T u, T u)=0$. On the contrary assume that $p(T u, T u)>0$, therefore from the inequality (2.1), we get

$$
\psi(p(u, u))+F(p(T u, T u)) \leqslant F(\phi(\mathbb{M}(u, u)))
$$

where

$$
\mathbb{M}(u, u)=\max \left\{p(u, u), p(u, T u), p(u, T u), \frac{1}{2}[p(u, T u)+p(u, T u)]\right\}=p(u, T u) .
$$

It follows from the above inequality that

$$
F(p(T u, T u)) \leqslant F(\phi(p(u, T u)))-\psi(p(u, u)),
$$

which implies

$$
\mathrm{F}(\mathrm{p}(\mathrm{Tu}, \mathrm{Tu}))<\mathrm{F}(\mathrm{p}(\mathrm{u}, \mathrm{Tu})),
$$

a contradiction. Thus, $p(T u, T u)=p(u, T u)=0$, which yields $u=T u$, that is, $u$ is a fixed point of $T$. Again, if $p(u, u)>0$, then by repeating the same process as mentioned above, one can easily obtain

$$
F(p(u, u)) \leqslant F(\phi(\mathbb{M}(u, u)))-\psi(p(u, u))
$$

which gives

$$
F(p(u, u))<F(p(u, u))
$$

a contradiction. Thus, $p(u, u)=0$. For uniqueness, let $v$ be another fixed point of $T$, that is, $T v=v$. If $p(u, v)>0$, then from (2.1), we can obtain

$$
\psi(p(u, v))+F(p(T u, T v))=\psi(p(u, v))+F(p(u, v)) \leqslant F(\phi(\mathbb{M}(u, v))),
$$

where

$$
\mathbb{M}(u, v)=\max \left\{p(u, v), p(u, T u), p(v, T v), \frac{1}{2}[p(u, T v)+p(v, T u)]\right\}=p(u, v) .
$$

Therefore, the above inequality turns into the following

$$
F(p(u, v)) \leqslant F(\phi(p(u, v)))-\psi(p(u, v)),
$$

which yields

$$
F(p(u, v))<F(p(u, v)),
$$

a contradiction. Hence we conclude that $p(u, v)=0$, that is, $u=v$. Thus the fixed point of $T$ is unique.

Here, we present an example which illustrates the usefulness of Theorem 2.2.

Example 2.3. Let $X=[0,1]$ and define $p: X \times X \rightarrow \mathbb{R}^{+}$by

$$
p(x, y)= \begin{cases}0, & \text { iff } x=y \\ \max \{x, y\}, & \text { otherwise }\end{cases}
$$

Without loss of generality, let us assume that $x \geqslant y$. It is obvious that $(X, p)$ is a 0 -complete partial metric space. Let $T: X \rightarrow X$ given by $T x=\frac{x+0.01}{10}$, then $T$ is continuous. Let $F(\alpha)=\log \alpha$, for all $\alpha \in \mathbb{R}^{+}$.

Define, $\psi:(0, \infty) \rightarrow(0, \infty)$ by $\psi(t)=\frac{1}{50(t+1)}$ and let $\phi:[0, \infty) \rightarrow[0, \infty)$ be given by $\phi(t)=\frac{98 t+1}{100}$. It is easy to see that $T$ is a Boyd-Wong type generalized $F-\psi$-contraction on $X$. In short we proceed as follows:

$$
\begin{aligned}
\text { L.H.S. } & =\psi(p(x, y))+F(p(T x, T y))=\frac{1}{50(x+1)}+\log \frac{x+0.01}{10} . \\
\text { R.H.S. } & =F\left(\phi\left(\max \left\{p(x, y), p\left(x, \frac{x+0.01}{10}\right), p\left(y, \frac{y+0.01}{10}\right), \frac{1}{2}\left[p\left(x, \frac{y+0.01}{10}\right)+p\left(y, \frac{x+0.01}{10}\right)\right]\right\}\right)\right) \\
& =\log (0.98 x+0.01) .
\end{aligned}
$$


Few choices of $x \in[0,1]$ give following:

$$
\begin{array}{ll}
\text { at } x=.1 & \text { L.H.S. }=-1.9404255<-0.9665762=\text { R.H.S. } \\
\text { at } x=.5 & \text { L.H.S. }=-1.2790964<-0.3010299=\text { R.H.S. } \\
\text { at } x=.8 & \text { L.H.S. }=-1.0804039<-0.1001794=\text { R.H.S. } \\
\text { at } x=1 & \text { L.H.S. }=-0.9856786<-0.0043648=\text { R.H.S.. }
\end{array}
$$

Thus, all the conditions of Theorem 2.2 are satisfied and $x=0.00111111111$ is the unique fixed point of $\mathrm{T}$ in $\mathrm{X}$. In Figure 1 red line represents $T$ and blue line represents $y=x$. It is clearly shown that there is unique fixed point of $\mathrm{T}$.

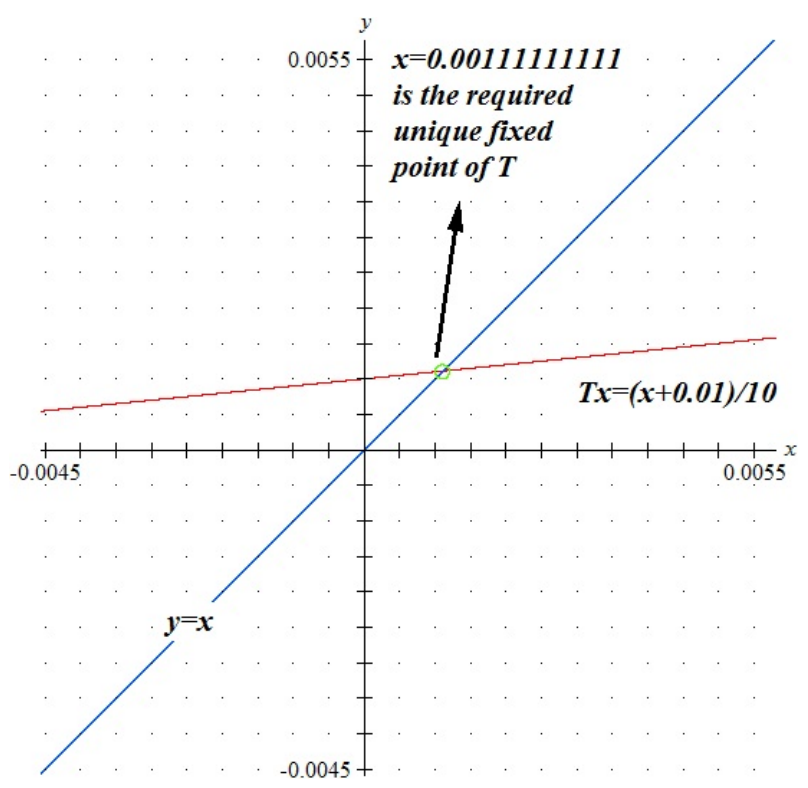

Figure 1: Plot of T.

In Figure 2 red curve represents RHS and green curve represents LHS of above example. Clearly red curve dominates green curve, which validates our inequality. Furthermore the values at some points are highlighted therein.

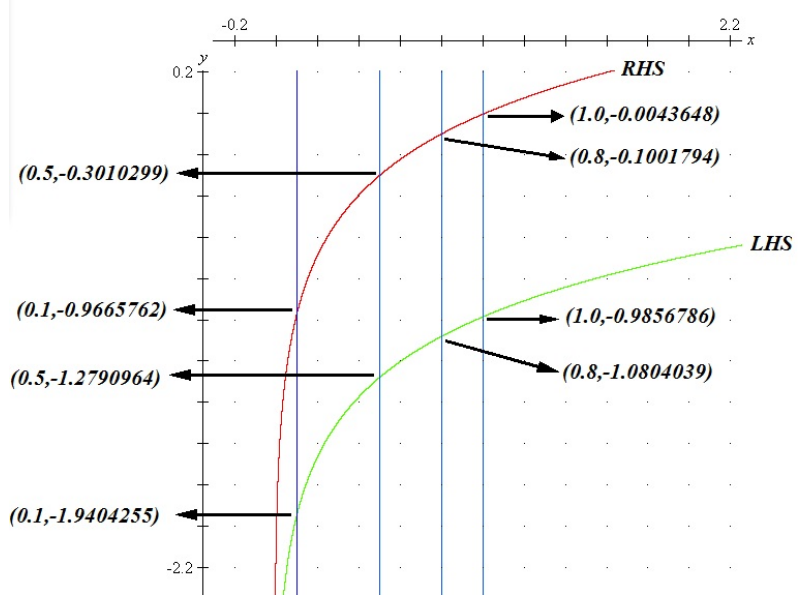

Figure 2: Validation of inequality. 
The following corollaries are immediate consequences of Theorem 2.2.

Corollary 2.4. Let $(\mathrm{X}, \mathrm{p})$ be a 0-complete partial metric space and $\mathrm{T}: \mathrm{X} \rightarrow \mathrm{X}$ be a self-map on $\mathrm{X}$. Suppose that there exist $\mathrm{F} \in \Delta_{\mathrm{F}}, \Psi \in \Psi$ and $\phi \in \Phi$ such that for all $\mathrm{x}, \mathrm{y} \in \mathrm{X}$ with $\mathrm{p}(\mathrm{Tx}, \mathrm{Ty})>0$,

$$
\psi(p(x, y))+F(p(T x, T y)) \leqslant F(\phi(\max \{p(x, y), p(x, T x), p(y, T y)\})) .
$$

Then $\mathrm{T}$ has a unique fixed point $\mathrm{u} \in \mathrm{X}$, moreover $\mathrm{p}(\mathrm{u}, \mathrm{u})=0$.

Corollary 2.5. Let $(\mathrm{X}, \mathrm{p})$ be a 0 -complete partial metric space. Let $\mathrm{T}: \mathrm{X} \rightarrow \mathrm{X}$ be a self-mapping on $\mathrm{X}$. Suppose that there exist $\mathrm{F} \in \Delta_{\mathrm{F}}, \psi \in \Psi$ and $\phi \in \Phi$ such that for all $\mathrm{x}, \mathrm{y} \in \mathrm{X}$ with $\mathrm{p}(\mathrm{Tx}, \mathrm{Ty})>0$,

$$
\psi(p(x, y))+F(p(T x, T y)) \leqslant F(\phi(p(x, y)))
$$

Then $\mathrm{T}$ has a unique fixed point $\mathrm{u} \in \mathrm{X}$, moreover $\mathrm{p}(\mathrm{u}, \mathrm{u})=0$.

Corollary 2.6. Let $(X, p)$ be a 0 -complete partial metric space and $\mathrm{T}: \mathrm{X} \rightarrow \mathrm{X}$ be an upper semi-continuous Boyd-Wong type generalized $\mathrm{F}-\psi$-contraction on $\mathrm{X}$. Then $\mathrm{T}$ has a unique fixed point $\mathrm{u} \in \mathrm{X}$, moreover $\mathrm{p}(\mathrm{u}, \mathrm{u})=0$.

Remark 2.7. Taking $\psi(t)=\tau>0$ and $\phi(t)=t$ in Theorem 2.2, we obtain the Corollary 13 of Shukla et al. [19] respectively.

Remark 2.8. If we take the function $\psi(t)=\tau>0$ and $\phi(t)=t$ in Corollary 2.5, then we find a F-contraction construction of the Banach fixed point theorem, obtained by Matthews [9] in the context of partial metric space.

Remark 2.9. Notice that by taking, $\psi(t)=\tau>0$ and $\phi(t)=t$ in Corollary 2.5, the result in Wardowski [21] can be also generalized for a 0-complete partial metric space. That is, we give a proper extension of the result in [21] in different aspect, more precisely, in the frame of 0-complete partial metric spaces.

2.2. Results on cyclic Boyd-Wong type generalized F- $\psi$-contractions in partial metric space

The succeeding definition and theorem will be needed to prove our next result.

Definition 2.10 ([8]). Let $X$ be a nonempty set, $m$ a positive integer, and $T: X \rightarrow X$ a mapping. $X=\bigcup_{i=1}^{m} A_{i}$ is said to be a cyclic representation of $X$ with respect to $T$, if

(i) $A_{i}, i=1,2, \cdots, m$ are nonempty closed sets;

(ii) $T\left(A_{1}\right) \subset A_{2}, \cdots, T\left(A_{m-1}\right) \subset T\left(A_{m}\right), T\left(A_{m}\right) \subset A_{1}$.

Theorem 2.11 ([4]). Let $(X, p)$ be a complete partial metric space on $X$ and let $m$ be a positive integer, $A_{1}, \cdots, A_{m}$ nonempty closed subsets of $\left(\mathrm{X}, \mathrm{d}_{\mathrm{p}}\right)$ and $\mathrm{Y}=\bigcup_{i=1}^{\mathrm{m}} \mathrm{A}_{i}$. Let $\mathrm{T}: \mathrm{Y} \rightarrow \mathrm{Y}$ be a mapping such that

(1) $\mathrm{Y}=\bigcup_{i=1}^{m} A_{i}$ is a cyclic representation of $\mathrm{Y}$ with respect to $\mathrm{T}$;

(2) there exists $\phi \in \Phi$ such that

$$
p(T x, T y) \leqslant \phi(\mathbb{M}(x, y))
$$

where

$$
\mathbb{M}(x, y)=\max \left\{p(x, y), p(x, T x), p(y, T y), \frac{1}{2}[p(x, T y)+p(y, T x)]\right\}
$$

for any $x \in A_{i}, y \in A_{i+1}, i=1,2, \cdots, m$, where $A_{m+1}=A_{1}$.

Then, $T$ has a unique fixed point $z \in \bigcap_{i=1}^{m} A_{i}$.

Utilizing the concept of Wardowski [21] and Aydi et al. [4], we introduce the following definition. 
Definition 2.12. Let $(X, p)$ be a partial metric space, $m$ be a positive integer, $A_{1}, A_{2}, \cdots, A_{m}$ nonempty closed subsets of $X$, and $Y=\bigcup_{i=1}^{m} A_{i}$. A mapping $T: Y \rightarrow Y$ is called a cyclic Boyd-Wong type generalized $\mathrm{F}-\Psi$-contraction, if

(1) $\bigcup_{i=1}^{m} A_{i}$ is a cyclic representation of $Y$ with respect to $T$;

(2) there exist $\psi \in \Psi, \phi \in \Phi$ and $F \in \Delta_{F}$ such that for all $p(T x, T y)>0$, the inequality (1.1) is satisfied, where the value of $M(x, y)$ is given by (1.2), for any $x \in A_{i}, y \in A_{i+1}, i=1,2, \cdots$, $m$, where $A_{m+1}=A_{1}$.

Theorem 2.13. Let $(\mathrm{X}, \mathrm{p})$ be a 0-complete partial metric space and $\mathrm{T}: \mathrm{Y} \rightarrow \mathrm{Y}$ be a cyclic Boyd-Wong type generalized $\mathrm{F}-\psi$-contraction on $\mathrm{X}$. If $\mathrm{T}$ is continuous, then $\mathrm{T}$ has a unique fixed point $z \in \bigcap_{i=1}^{\mathrm{m}} A_{i}$.

Proof. Let $x_{0} \in Y=\bigcup_{i=1}^{m} A_{i}$. Consider the Picard iteration $\left\{x_{n}\right\}$ be given by $T x_{n}=x_{n+1}$ for $n \in \mathbb{N}^{*}$. If there exists $n_{0} \in \mathbb{N}^{*}$ such that $x_{n_{0}}=x_{n_{0}+1}$, then $x_{n_{0}}$ is the required fixed point of $T$ and the proof is completed.

Consequently, suppose that $x_{n} \neq x_{n+1}$ or $p\left(T x_{n-1}, T x_{n}\right)>0$ for all $n \in \mathbb{N}^{*}$. Also keep in mind that $Y=\bigcup_{i=1}^{m} A_{i}$, thus for each $n \in \mathbb{N}^{*}$, there exists $i_{n} \in\{1,2, \cdots, m\}$ such that $x \in A_{i}$ and $x_{n+1}=T x_{n} \in$ $T\left(A_{i_{n}}\right) \subseteq A_{i_{n}+1}$. It follows from inequality (2.1) that

$$
\begin{aligned}
\psi\left(p\left(x_{n-1}, x_{n}\right)\right)+F\left(p\left(x_{n}, x_{n+1}\right)\right) & =\psi\left(p\left(x_{n-1}, x_{n}\right)\right)+F\left(p\left(T x_{n-1}, T x_{n}\right)\right) \\
& \leqslant F\left(\phi\left(\mathbb{M}\left(x_{n-1}, x_{n}\right)\right)\right) .
\end{aligned}
$$

On the similar lines of done in Theorem 2.2, one can easily show that

$$
\lim _{n \rightarrow \infty} F\left(p\left(x_{n}, x_{n+1}\right)\right)=-\infty \Longleftrightarrow \lim _{n \rightarrow \infty} p\left(x_{n}, x_{n+1}\right)=0 .
$$

Now, we claim that $\left\{x_{n}\right\}_{n=1}^{\infty}$ is a Cauchy sequence in the partial metric space $\left(Y=\bigcup_{i=1}^{m} A_{i}, p\right)$. On the contrary assume that $\left\{x_{n}\right\}_{\mathfrak{n}=1}^{\infty}$ is not a Cauchy sequence in $(Y, p)$. Then there exists $\epsilon>0$ for which we can find two sub-sequences $\left\{x_{n(k)}\right\}$ and $\left\{x_{m(k)}\right\}$ of $\left\{x_{n}\right\}_{n=1}^{\infty}$ such that, $n(k)$ is the smallest index for which $n(k)>m(k) \geqslant k$ and

$$
p\left(x_{m(k)}, x_{n(k)}\right) \geqslant \epsilon .
$$

Again, in a similar way as in the proof of Theorem 2.2, one can show that

$$
\lim _{k \rightarrow \infty} p\left(x_{m(k)}, x_{n(k)}\right)=\epsilon .
$$

Moreover,

$$
\begin{aligned}
& \lim _{k \rightarrow \infty} p\left(x_{m(k)}, x_{n(k)+1}\right)=\epsilon, \\
& \lim _{k \rightarrow \infty} p\left(x_{m(k)+1}, x_{n(k)}\right)=\epsilon,
\end{aligned}
$$

and

$$
\lim _{k \rightarrow \infty} p\left(x_{m(k)+1}, x_{n}(k)+1\right)=\epsilon .
$$

On the other hand, for all $k$ we can find $j(k)$, where $0 \leqslant j(k) \leqslant p$ such that $n(k)-m(k)+j(k) \equiv 1(p)$, which yields $x_{m(k)-j(k)}$ (for $k$ large enough, $m(k)>j(k)$ ) and $x_{n(k)}$ lies in the different adjacently labeled sets $A_{i}$ and $A_{i+1}$ for certain $i=1,2, \cdots, p$.

From inequalities (2.15) and (2.16) we have

$$
\lim _{k \rightarrow \infty} p\left(x_{n(k)}, x_{m(k)-j(k)}\right)=\lim _{k \rightarrow \infty} p\left(x_{n(k)+1}, x_{m(k)-j(k)}\right)=\epsilon,
$$


and

$$
\lim _{k \rightarrow \infty} p\left(x_{n(k)}, x_{m(k)-j(k)+1}\right)=\lim _{k \rightarrow \infty} p\left(x_{n(k)+1}, x_{m(k)-j(k)+1}\right)=\epsilon .
$$

As $p\left(T x_{n(k)}, T x_{m(k)-j(k)}\right)>0$, by the contractive condition (1.1) we acquire

$$
\begin{aligned}
\psi\left(p\left(x_{n(k)}, x_{m(k)-j(k)}\right)\right)+F\left(p\left(x_{n(k)+1}, x_{m(k)-j(k)+1}\right)\right)= & \psi\left(p\left(x_{n(k)}, x_{m(k)-j(k)}\right)\right) \\
& +F\left(p\left(T x_{n(k)}, T x_{m(k)-j(k)}\right)\right) \\
\leqslant & F\left(\phi\left(M\left(x_{n(k)}, x_{m(k)-j(k)}\right)\right)\right) .
\end{aligned}
$$

It follows from the property of $\psi$, that

$$
\begin{aligned}
F\left(p\left(x_{n(k)+1}, x_{m(k)-j(k)+1}\right)\right) & \leqslant F\left(\phi\left(\mathbb{M}\left(x_{n(k)}, x_{m(k)-j(k)}\right)\right)\right)-\psi\left(p\left(x_{n(k)}, x_{m(k)-j(k)}\right)\right) \\
& \leqslant F\left(\phi\left(\mathbb{M}\left(x_{n(k)}, x_{m(k)-j(k)}\right)\right)\right) .
\end{aligned}
$$

By the definition of $M(x, y)$ and taking into account (2.17), we find that

$$
\lim _{k \rightarrow \infty} \mathbb{M}\left(x_{n(k)}, x_{m(k)-j(k)}\right)=\epsilon .
$$

Indeed

$$
\begin{aligned}
\mathbb{M}\left(x_{n(k)}, x_{m(k)-j(k)}\right)=\max \{ & p\left(x_{n(k)}, x_{m(k)-j(k)}\right), p\left(x_{n(k)}, x_{n(k)+1}\right), \\
& p\left(x_{m(k)-j(k)}, x_{m(k)-j(k)+1),}\right. \\
& \left.\frac{1}{2}\left[p\left(x_{n(k)}, x_{m(k)-j(k)}+1\right)+p\left(x_{n(k)+1}, x_{m(k)-j(k)}\right)\right]\right\} .
\end{aligned}
$$

Letting $k \rightarrow \infty$ in (2.18) and using (2.17), (2.19), property (F3') together with the upper semi-continuity of $\phi$, we get $F(\epsilon) \leqslant F(\phi(\epsilon))<F(\epsilon)$ a contradiction. Thus $\left\{x_{n}\right\}_{\mathfrak{n}=1}^{\infty}$ is a Cauchy sequence in the partial metric space $(Y, p)$. By Lemma $1.4\left\{x_{n}\right\}_{n=1}^{\infty}$ is a Cauchy sequence in the complete metric space $(Y=$ $\left.\bigcup_{i=1}^{m} A_{i}, d_{p}\right)$, which yields, there exists $u \in Y$ such that $u=\lim _{n \rightarrow \infty} x_{n} \in\left(Y, d_{p}\right)$.

It is clear that the sequence $\left\{x_{n}\right\}_{\mathfrak{n} \in \mathbb{N}}$ has an infinite number of terms in each $A_{i}, i=1,2, \cdots, m$, as $\left(Y, d_{p}\right)$ is complete, hence from each $A_{i}, i=1,2, \cdots, m$, one can easily construct a sub-sequence of $\left\{x_{n}\right\}$ that converges to $u$. $A_{i}, i=1,2, \cdots, m$ are closed in $\left(Y, d_{p}\right)$, therefore $u \in \bigcap_{i=1}^{m} A_{i}$. Hence $\bigcap_{i=1}^{m} A_{i}=A \neq$ $\phi$.

Clearly, $A$ is closed in $\left(Y, d_{p}\right)$ and it is a complete subspace of $\left(Y, d_{p}\right)$ and it follows that $(A, p)$ is a complete partial metric space. Consider the restriction of $T$ on $A$, i.e., $T_{/ A}: A \rightarrow A$. Then $T_{/ A}$ satisfies all the conditions of Theorem 2.2. Hence, $T_{/ A}$ has a unique fixed point.

Following example authenticates the effectiveness of the hypotheses and degree of generality of Theorem 2.13.

Example 2.14. Let $X=[0,1]$. Let the partial metric $p$ on $X$ is described by

$$
p(x, y)= \begin{cases}0, & \text { iff } x=y \\ \max \{x, y\}, & \text { otherwise }\end{cases}
$$

Without loss of generality, let us assume that $x \geqslant y$. It is easy to see that $(X, p)$ is a 0 -complete partial metric space. Set $A_{1}=[0,0.25], A_{2}=[0,0.5]$ and $Y=A_{1} \cup A_{2}=[0,0.5]$. Also define the mapping $T$ by $\mathrm{T} x=\frac{\mathrm{x}+0.04}{20}$.

Note that, $\mathrm{T}\left(\mathrm{A}_{1}\right)=\mathrm{T}([0,0.25])=[0.002,0.0145] \subset[0,0.5]=A_{2}$ and $\mathrm{T}\left(\mathrm{A}_{2}\right)=\mathrm{T}([0,0.5])=[0.002,0.027] \subset$ $[0,0.25]=A_{1}$. Hence $Y=A_{1} \cup A_{2}$ has a cyclic representation of $T$.

Clearly $T$ is continuous and let $F(\alpha)=\log \alpha+\alpha$ for all $\alpha \in \mathbb{R}^{+}$. 
Take, $\psi:(0, \infty) \rightarrow(0, \infty)$ by $\psi(t)=\frac{1}{100(t+1)}$ and let $\phi:[0, \infty) \rightarrow[0, \infty)$ be given by $\phi(t)=\frac{120 t+2}{123}$. It is easy to see that $\mathrm{T}$ satisfies condition (1.1). Succinctly we progress as follows:

$$
\begin{aligned}
\text { L.H.S. } & =\psi(p(x, y))+F(p(T x, T y))=\frac{1}{100(x+1)}+\log \frac{x+0.04}{20}+\frac{x+0.04}{20} . \\
\text { R.H.S. } & =F\left(\phi\left(\max \left\{p(x, y), p\left(x, \frac{x+0.04}{20}\right), p\left(y, \frac{y+0.04}{20}\right), \frac{1}{2}\left[p\left(x, \frac{y+0.04}{20}\right)+p\left(y, \frac{x+0.04}{20}\right)\right]\right\}\right)\right) \\
& =\log \frac{120 x+2}{123}+\frac{120 x+2}{123} .
\end{aligned}
$$

Some choices of $x \in[0,0.5]$ give as follows:

$$
\begin{aligned}
\text { at } x=.1 & \text { L.H.S. }=-2.1388110<-0.8299559=\text { R.H.S. } \\
\text { at } y=.2 & \text { L.H.S. }=-1.9004854<-0.4635496=\text { R.H.S. } \\
\text { at } y=.3 & \text { L.H.S. }=-1.7448587<-0.2011784=\text { R.H.S. } \\
\text { at } y=.4 & \text { L.H.S. }=-1.6284344<0.0155689=\text { R.H.S. } \\
\text { at } y=.5 & \text { L.H.S. }=-1.5349695<0.2065516=\text { R.H.S.. }
\end{aligned}
$$

Hence, all the hypotheses of Theorem 2.13 are satisfied and $x=0.00210526316$ is the unique fixed point of $\mathrm{T}$ in $\mathrm{X}$.

In Figure 3 red curve represents $T$ and blue line represents $T x=x$. It is clearly shown that there is unique fixed point of $\mathrm{T}$.

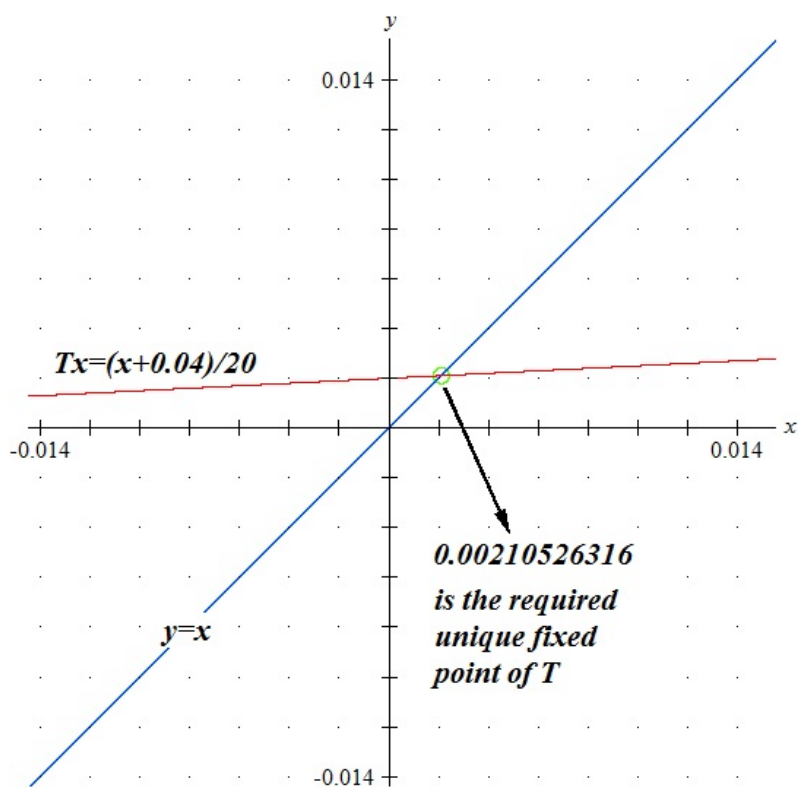

Figure 3: Plot of T.

In Figure 4 red curve represents RHS and green curve represents LHS of above example. Clearly red curve dominates green curve, which validates our inequality. Furthermore the values at some points are highlighted therein.

Remark 2.15. As every partial metric space is a metric space with zero self-distance. Thus if we replace partial metric $p$ by a metric $d$ in Theorem 2.2 and Theorem 2.13, then we get the fixed point theorems for Boyd-Wong type generalized F- $\psi$-contraction and cyclic Boyd-Wong type generalized F- $\psi$-contraction in complete metric space, respectively. Also our theorems generalize and extend the following theorems in the following aspects. 
(i) Taking $\psi(t)=\tau>0$ and $\phi(t)=t$ in Theorem 2.2, then the result in Theorem 2.2 is a natural generalization of Ciric type Generalized F-contractions due to G. Minak et al. [10] and F-weak contractions due to Wardowski et al. [22].

On the other hand, we see that G. Minak et al. [10] claimed the definition as Ciric type generalized F-contraction while Wardowski et al. [22] claimed F-weak contraction in their note.

(ii) Taking $\psi(t)=\tau>0$ and $\phi(t)=t$ in Theorem 2.13, we generalize the result of Kirk et al. [8].

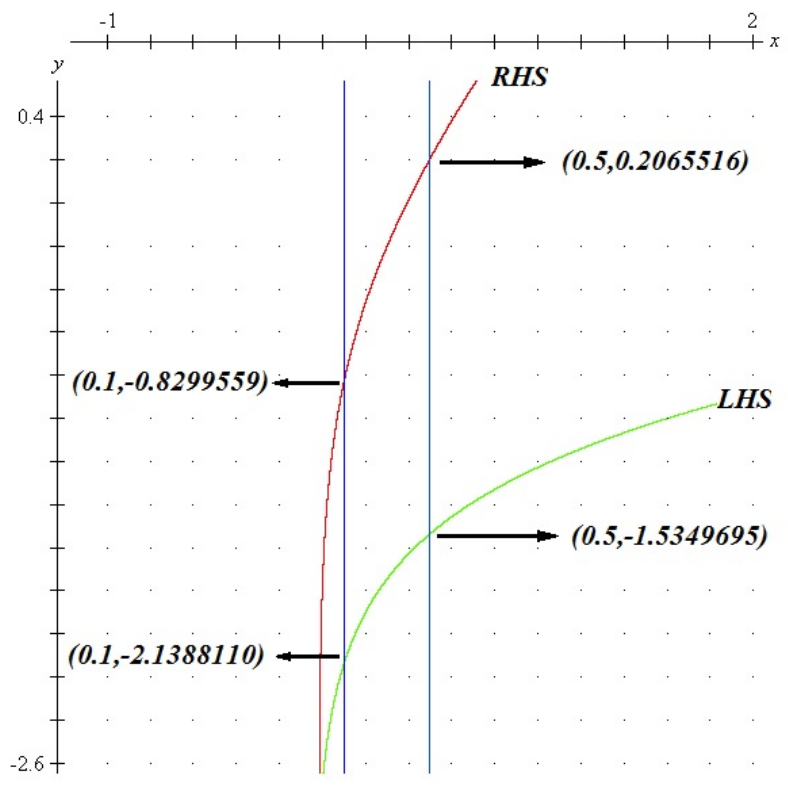

Figure 4: Validation of inequality.

Remark 2.16. By introducing F-contraction, Wardowski [21] stated the modified version of Bananch contraction principle. In [21], Wardowski proved that the Bananch contractions are particular case of Fcontractions. Meanwhile, the author presented some F-contractions which are not Banach contractions.

In view of aforesaid, we obtained generalized, extended new version of main results of Romaguera [16] and Aydi et al. [4] by launching Theorem 2.2 and Theorem 2.13 in 0-complete partial metric spaces for F-contraction, respectively.

Remark 2.17. Notice that we get the same result, if we replace 0-complete partial metric space with complete partial metric space in Theorem 2.2 and Theroem 2.13.

\section{Some applications}

\subsection{Application to a dynamical programming}

Basically a dynamical process consists of:

(i) a state space, which is the set of the initial state, actions, and transition of the process;

(ii) a decision space, which is the set of possible actions that are allowed for the process.

The dynamic processing gives fruitful tools for mathematical optimization and computer programming. In aforesaid system, the problem of dynamic programming associated to multistage process reduces to the problem of solving the functional equation

$$
q(x)=\sup _{y \in D}\{f(x, y)+G(x, y, q(\rho(x, y)))\}, \quad x \in W,
$$


where $\rho: W \times D \rightarrow W, f: W \times D \rightarrow \mathbb{R}$ and $G: W \times D \times \mathbb{R} \rightarrow \mathbb{R}$. We suppose that $W \subseteq U$ is a state space, $\mathrm{D} \subseteq \mathrm{V}$ is a decision space, $\mathrm{U}$ and $\mathrm{V}$ are Banach spaces. Let $\mathrm{B}(\mathrm{W})$ denote the set of all bounded real valued functions on $W$ and for an arbitrary $h \in B(W)$, define $\|h\|=\sup _{x \in W}|h(x)|$. Clearly, the pair $(B(W),\|\|$. with the metric $\mathrm{d}$ defined by

$$
d(h, k)=\sup _{x \in W}|h(x)-k(x)|,
$$

for all $h, k \in B(W)$ is a Banach space. Precisely, the convergence in the space $B(W)$ with respect to $\|$. is uniform and thus, if we consider a Cauchy sequence $\left\{h_{n}\right\}$ in $B(W)$, then $\left\{h_{n}\right\}$ converges uniformly to a function, say $h^{*}$, that is bounded and so $h^{*} \in B(W)$. Now for all $h, k \in B(W), x \in W$ and $b>0$, we consider the partial metric $p$ defined by

$$
p(h, k)=d(h, k)+b=\sup _{x \in W}|h(x)-k(x)|+b,
$$

and we also define $\mathrm{T}: \mathrm{B}(\mathrm{W}) \rightarrow \mathrm{B}(\mathrm{W})$ by

$$
T(h)(x)=\sup _{y \in D}\{f(x, y)+G(x, y, h(\rho(x, y)))\},
$$

for all $h \in B(W)$ and $x \in W$. Obviously, $T$ is well-defined, if the functions $f$ and $G$ are bounded.

The subsequent result is followed:

Theorem 3.1. Let $\mathrm{T}$ be an upper semi-continuous operator defined by (3.2) and suppose that the following conditions hold:

(1) $\mathrm{G}: \mathrm{W} \times \mathrm{D} \times \mathbb{R} \rightarrow \mathbb{R}$ and $\mathrm{f}: \mathrm{W} \times \mathrm{D} \rightarrow \mathbb{R}$ are continuous and bounded;

(2) there exist a decreasing function $\psi:(0, \infty) \rightarrow(0, \infty)$ and a function $\phi:[0, \infty) \rightarrow[0, \infty)$ such that

(i) $\phi$ is upper semi continuous and $\phi(t)<t$ for each $t>0$;

(ii)

with

$$
|G(x, y, h(x))-G(x, y, k(x))| \leqslant e^{-\psi(|h-k|+b)} \phi(\mathbb{M}(h, k)),
$$

$$
\mathbb{M}(h, k)=\max \left\{p(h, k), p(h, T(h)), p(k, T(k)), \frac{1}{2}[p(h, T(k))+p(k, T(h))]\right\}
$$

for all $h, k \in \mathrm{B}(\mathrm{W}), \mathrm{x} \in \mathrm{W}$ and $\mathrm{y} \in \mathrm{D}$.

Then the functional equation (3.1) has a bounded solution.

Proof. Let $\lambda$ be an arbitrary positive number, $x \in W$ and $h_{1}, h_{2} \in B(W)$, then there exist $y_{1}, y_{2} \in D$ such that

$$
\begin{gathered}
T\left(h_{1}\right)(x)<f\left(x, y_{1}\right)+G\left(x, y_{1}, h_{1}\left(\rho\left(x, y_{1}\right)\right)\right)-b+\lambda, \\
T\left(h_{2}\right)(x)<f\left(x, y_{2}\right)+G\left(x, y_{2}, h_{2}\left(\rho\left(x, y_{2}\right)\right)\right)-b+\lambda, \\
T\left(h_{1}\right)(x) \geqslant f\left(x, y_{2}\right)+G\left(x, y_{1}, h_{1}\left(\rho\left(x, y_{1}\right)\right)\right), \\
T\left(h_{2}\right)(x) \geqslant f\left(x, y_{1}\right)+G\left(x, y_{2}, h_{2}\left(\rho\left(x, y_{2}\right)\right)\right),
\end{gathered}
$$

which yields

$$
\begin{aligned}
T\left(h_{1}\right)(x)-T\left(h_{2}\right)(x) & <G\left(x, y_{1}, h_{1}\left(\rho\left(x, y_{1}\right)\right)\right)-G\left(x, y_{2}, h_{2}\left(\rho\left(x, y_{2}\right)\right)\right)-b+\lambda \\
& \leqslant\left|G\left(x, y_{1}, h_{1}\left(\rho\left(x, y_{1}\right)\right)\right)-G\left(x, y_{2}, h_{2}\left(\rho\left(x, y_{2}\right)\right)\right)\right|-b+\lambda \\
& \leqslant e^{-\psi\left(\left|h_{1}-h_{2}\right|+b\right)} \phi\left(\mathbb{M}\left(h_{1}, h_{2}\right)\right)-b+\lambda .
\end{aligned}
$$

In a same manner, we arrive at

$$
\mathrm{T}\left(\mathrm{h}_{2}\right)(x)-\mathrm{T}\left(\mathrm{h}_{1}\right)(x) \leqslant e^{-\psi\left(\left|h_{1}-h_{2}\right|+b\right)} \phi\left(\mathbb{M}\left(h_{1}, h_{2}\right)\right)-b+\lambda .
$$


Therefore

$$
\begin{gathered}
\left|T\left(h_{1}\right)(x)-T\left(h_{2}\right)(x)\right| \leqslant e^{-\psi\left(\left|h_{1}-h_{2}\right|+b\right)} \phi\left(\mathbb{M}\left(h_{1}, h_{2}\right)\right)-b+\lambda, \\
p\left(T\left(h_{1}\right), T\left(h_{2}\right)\right) \leqslant e^{-\psi\left(p\left(h_{1}, h_{2}\right)\right)} \phi\left(\mathbb{M}\left(h_{1}, h_{2}\right)\right)+\lambda .
\end{gathered}
$$

Since the above inequality does not depend on $x \in W$ and $\lambda>0$ is taken arbitrary, then we conclude that

$$
p\left(T\left(h_{1}\right), T\left(h_{2}\right)\right) \leqslant e^{-\psi\left(p\left(h_{1}, h_{2}\right)\right)} \phi\left(\mathbb{M}\left(h_{1}, h_{2}\right)\right),
$$

for each $x \in W$. Consequently, by passing to logarithms, one can get

$$
\psi\left(p\left(h_{1}, h_{2}\right)\right)+\log \left(p\left(T\left(h_{1}\right), T\left(h_{2}\right)\right)\right) \leqslant \log \phi\left(\left(\mathbb{M}\left(h_{1}, h_{2}\right)\right)\right) .
$$

This turns up to

$$
\psi\left(p\left(h_{1}, h_{2}\right)\right)+F\left(p\left(T\left(h_{1}\right), T\left(h_{2}\right)\right)\right) \leqslant F\left(\phi\left(\mathbb{M}\left(h_{1}, h_{2}\right)\right)\right),
$$

for $F(t)=\log t, t>0$. Thus we conclude that $T$ is an F-contraction. Due to Corollary 2.6, $T$ has a fixed point $h^{*} \in B(W)$, that is $h^{*}$ is a bounded solution of the functional equation (3.1).

Remark 3.2. We also suggest some change of notation given by Sgroi et al. [18]. Notice that, in the context of equation (9), authors used the notation $\tau$, where $\tau: W \times D \rightarrow W$ and in Theorem 5.1, they used the same notation $\tau$, where $\tau \in \mathbb{R}^{+}$which is puzzling, so one need to replace notation $\tau$ in (9), to avoid any confusion among researchers.

\subsection{Application to an integral inequality}

Let $\Lambda$ denote the set of functions $\mu:[0, \infty) \rightarrow[0, \infty)$ satisfying the following hypotheses:

(i) $\mu$ is a Lebesgue integrable mapping on each compact of $[0, \infty)$;

(ii) for every $\epsilon>0$, we have $\int_{0}^{\epsilon} \mu(t) d t>0$.

Theorem 3.3. Let $(\mathrm{X}, \mathrm{p})$ be a 0 -complete partial metric space and $\mathrm{T}$ be a mapping on $\mathrm{X}$. For all $\mathrm{X}, \mathrm{y} \in \mathrm{X}$ with $\int_{0}^{p(T x, T y)} \mu(t) d t>0$ we have

$$
\int_{0}^{\mathfrak{p}(x, y)} \mu_{1}(t) d t+F\left(\int_{0}^{\mathfrak{p}(T x, T y)} \mu(t) d t\right) \leqslant F\left(\int_{0}^{\max \left\{p(x, y), p(x, T x), p(y, T y), \frac{1}{2}[p(x, T y)+p(y, T x)]\right\}} \mu_{2}(t) d t\right),
$$

where $\mu, \mu_{1}, \mu_{2} \in \Lambda$. Then $\mathrm{T}$ has a unique fixed point.

\section{Conclusions}

In our finding, recognizing the concept of F-contraction due to Wardowski [21] and Piri and Kumam [14], some unique fixed point theorems for Boyd-Wong and cyclic Boyd-Wong type generalized F- $\psi$ contractions in 0-complete partial metric space are established. The applications and illustrative examples show the high degree of reliability to other authors to generalize and improve these results for future research.

\section{Acknowledgment}

This project was supported by the Theoretical and Computational Science (TaCS) Center under Computational and Applied Science for Smart Innovation Research Cluster (CLASSIC), Faculty of Science, KMUTT. Moreover, this research work was partially financially supported by King Mongkuts University of Technology North Bangkok. Contract No. KMUTNB-60-ART-086. 


\section{References}

[1] M. Abbas, T. Nazir, S. Romaguera, Fixed point results for generalized cyclic contraction mappings in partial metric spaces, Rev. R. Acad. Cienc. Exactas Fís. Nat. Ser. A Math. RACSAM, 106 (2012), 287-297. 1

[2] M. Akram, W. Shamaila, A coincident point and common fixed point theorem for weakly compatible mappings in partial metric spaces, J. Nonlinear Sci. Appl., 8 (2015), 184-192. 1

[3] I. Altun, F. Sola, H. Simsek, Generalized contractions on partial metric spaces, Topology Appl., 157 (2010), $2778-2785$. 1

[4] H. Aydi, E. Karapınar, A fixed point result for Boyd-Wong cyclic contractions in partial metric spaces, Int. J. Math. Math. Sci., 2012 (2012), 11 pages. 1, 1, 2.11, 2.2, 2.16

[5] D. W. Boyd , J. S. W. Wong, On nonlinear contractions, Proc. Amer. Math. Soc., 20 (1969), 458-464. 1, 1

[6] X.-J. Huang, Y.-Y. Li, C.-X. Zhu, Multivalued f-weakly Picard mappings on partial metric spaces, J. Nonlinear Sci. Appl., 8 (2015), 1234-1244. 1

[7] A. Hussain, M. Arshad, S. U. Khan, $\tau$-Generalization of Fixed Point Results for F-Contractions, Bangmod Int. J. Math. \& Comp. Sci., 1 (2015), 136-146. 1

[8] W. A. Kirk, P. S. Srinivasan, P. Veeramani, Fixed points for mappings satisfying cyclical contractive conditions, Fixed Point Theory, 4 (2003), 79-89. 2.10, 2.15

[9] S. G. Matthews, Partial metric topology, Papers on general topology and applications, Flushing, NY, (1992), 183-197, Ann. New York Acad. Sci., New York Acad. Sci., New York, 728 (1994). 1, 1.1, 1.2, 1.3, 1.4, 1.5, 2.8

[10] G. Minak, A. Helvac1, I. Altun, Ćiriţype generalized F-contractions on complete metric spaces and fixed point results, Filomat, 28 (2014), 1143-1151. 2.15

[11] A. Nastasi, P. Vetro, Fixed point results on metric and partial metric spaces via simulation functions, J. Nonlinear Sci. Appl., 8 (2015), 1059-1069. 1

[12] S. Oltra, O. Valero, Banach's fixed point theorem for partial metric spaces, Rend. Istit. Mat. Univ. Trieste, 36 (2004), 17-26. 1, 1.3, 1.4

[13] D. Paesano, P. Vetro, Suzuki's type characterizations of completeness for partial metric spaces and fixed points for partially ordered metric spaces, Topology Appl., 159 (2012), 911-920. 1.6

[14] H. Piri, P. Kumam, Some fixed point theorems concerning F-contraction in complete metric spaces, Fixed Point Theory Appl., 2014 (2014), 11 pages. 1, 1, 4

[15] S. Romaguera, A Kirk type characterization of completeness for partial metric spaces, Fixed Point Theory Appl., 2010 (2010), 6 pages. $1,1.3,1.4$

[16] S. Romaguera, Fixed point theorems for generalized contractions on partial metric spaces, Topology Appl., 159 (2012), 194-199. 1, 1, 1, 2.16

[17] N.-A. Secelean, Iterated function systems consisting of F-contractions, Fixed Point Theory Appl., 2013 (2013), 13 pages. 1,1

[18] M. Sgroi, C. Vetro, Multi-valued F-contractions and the solution of certain functional and integral equations, Filomat, 27 (2013), 1259-1268. 3.2

[19] S. Shukla, S. Radenović, Some common fixed point theorems for F-contraction type mappings on 0-complete partial metric spaces, J. Math., 2013 (2013), 7 pages. 2.7

[20] O. Valero, On Banach fixed point theorems for partial metric spaces, Appl. Gen. Topol., 6 (2005), 229-240. 1

[21] D. Wardowski, Fixed points of a new type of contractive mappings in complete metric spaces, Fixed Point Theory Appl., 2012 (2012), 6 pages. 1, 1, 1, 1, 2.9, 2.2, 2.16, 4

[22] D. Wardowski, N. Van Dung, Fixed points of F-weak contractions on complete metric spaces, Demonstr. Math., 47 (2014), 146-155. 2.15 